\title{
What is (not) wrong with scalar gravity?
}

\author{
Domenico Giulini \\ Max-Planck-Institute for Gravitational Physics, Albert-Einstein-Institute, Am Mühlenberg 1, \\ D-14476 Golm, Germany
}

Received 14 December 2006; received in revised form 30 August 2007

\begin{abstract}
On his way to General Relativity, Einstein gave several arguments as to why a special-relativistic theory of gravity based on a massless scalar field could be ruled out merely on grounds of theoretical considerations. We re-investigate his two main arguments, which relate to energy conservation and some form of the principle of the universality of free fall. We find such a theory-based a priori abandonment not to be justified. Rather, the theory seems formally perfectly viable, though in clear contradiction with (later) experiments.
\end{abstract}

(C) 2007 Elsevier Ltd. All rights reserved.

Keywords: General relativity; Gravity; Special relativity

When citing this paper, please use the full journal title Studies in History and Philosophy of Modern Physics

\section{Introduction}

General Relativity (henceforth 'GR') differs markedly in many structural aspects from all other theories of fundamental interactions, which are all formulated as Poincaré invariant theories in the framework of Special Relativity (henceforth 'SR'). The characterisation of this difference has been a central theme not only for physicists, but also for philosophers and historians of science. Einstein himself emphasised in later (1933)

E-mail address: domenico.giulini@aei.mpg.de 
recollections the importance of his failure to formulate a viable special-relativistic theory of gravity for the understanding of the genesis of GR.

Any attempt to give such a characterisation should clearly include a precise description of the constraints that prevent gravity from also fitting into the framework of SR. In modern terminology, a natural way to proceed would be to consider fields according to mass and spin, ${ }^{1}$ discuss their possible equations, the inner consistency of the mathematical schemes so obtained, and finally their experimental consequences. Since gravity is a classical, macroscopically observable, and long-ranged field, one usually assumes right at the beginning the spin to be integral and the mass parameter to be zero. The first thing to consider would therefore be a massless scalar field. What goes wrong with such a theory?

When one investigates this question, anticipating that something does indeed go wrong, one should clearly distinguish between the following two types of reasoning:

1. The theory is internally inconsistent. In a trivial sense this may mean that it is mathematically contradictory, in which case this is the end of the story. On a more sophisticated level it might also mean that the theory violates accepted fundamental physical principles, like, e.g., that of energy conservation, without being plainly mathematically contradictory.

2. The theory is formally consistent and in accord with basic physical principles. However, it is refuted by experiments.

Note that, generically, it does not make much sense to claim both shortcomings simultaneously, since 'predictions' of inconsistent theories should not be trusted. The question to be addressed here is whether special-relativistic theories of scalar gravity fall under the first category, i.e. whether they can be refuted on the basis of formal arguments alone without reference to specific experiments.

Many people think that it can, following A. Einstein who accused scalar theories to

(a) violate some form of the principle of universality of free fall,

(b) violate energy conservation.

The purpose of this paper is to investigate these statements in detail. We will proceed by the standard (Lagrangian) methods of modern field theory and take what we perceive as the obvious route when working from first principles.

\section{Historical background}

As already stressed, the abandonment of scalar theories of gravity by Einstein is intimately linked with the birth of GR, in particular with his conviction that general covariance must replace the principle of relativity as used in SR.

I will focus on two historical sources in which Einstein complains about scalar gravity not being adequate. One is his joint paper with Marcel Grossman on the so-called 'Entwurf Theory' (Klein et al., 1995, Doc. 13, henceforth called the 'Entwurf Paper'), of which Grossmann wrote the 'mathematical part' and Einstein the 'physical part'. Einstein

\footnotetext{
${ }^{1}$ Mass and spin are the eigenvalues of the so-called Casimir operators of the Poincare group, that label its irreducible representations.
} 
finished with Section 7, whose title asks: 'Can the gravitational field be reduced to a scalar?' (German original: 'Kann das Gravitationsfeld auf einen Skalar zurückgeführt werden?'). In this paragraph he presented a Gedankenexperiment-based argument which allegedly shows that any special-relativistic scalar theory of gravity, in which the gravitational field couples exclusively to the matter via the trace of its energy-momentum tensor, necessarily violates energy conservation and is hence physically inconsistent. This he presented as plausibility argument why gravity has to be described by a more complex quantity, like the $g_{\mu v}$ of the Entwurf Paper, where he and Grossmann consider 'generally covariant' equations for the first time. After having presented his argument, he ends Section 7 (and his contribution) with the following sentences, expressing his conviction in the validity of the principle of general covariance:

Einstein Quote 1. Ich muß freilich zugeben, daß für mich das wirksamste Argument dafür, daß eine derartige Theorie [eine skalare Gravitationstheorie] zu verwerfen sei, auf der Überzeugung beruht, daß die Relativität nicht nur orthogonalen linearen Substitutionen gegenüber besteht, sondern einer viel weitere Substitutionsgruppe gegenüber. Aber wir sind schon desshalb nicht berechtigt, dieses Argument geltend zu machen, weil wir nicht imstande waren, die (allgemeinste) Substitutionsgruppe ausfindig zu machen, welche zu unseren Gravitationsgleichungen gehört. ${ }^{2}$ (Klein et al., 1995, Doc. 13, p. 323)

The other source where Einstein reports in more detail on his earlier experiences with scalar gravity is his manuscript entitled 'Einiges über die Entstehung der Allgemeinen Relativitätstheorie', dated June 20th 1933, reprinted in (Einstein, 2005, pp. 176-193). There he describes in words (no formulae are given) how the 'obvious' special-relativistic generalisation of the Poisson equation,

$$
\Delta \Phi=4 \pi G \rho,
$$

together with a (slightly less obvious) special-relativistic generalisation of the equation of motion,

$$
\frac{\mathrm{d}^{2} \vec{x}(t)}{\mathrm{d} t^{2}}=-\vec{\nabla} \Phi(\vec{x}(t))
$$

lead to a theory in which the vertical acceleration of a test particle in a static homogeneous vertical gravitational field depends on its initial horizontal velocity and also on its internal energy content. In his own words:

Einstein Quote 2. Solche Untersuchungen führten aber zu einem Ergebnis, das mich in hohem Maß mißtrauisch machte. Gemäß der klassischen Mechanik ist nämlich die Vertikalbeschleunigung eines Körpers im vertikalen Schwerefeld von der Horizontalkomponente der Geschwindigkeit unabhängig. Hiermit hängt es zusammmen, daß die Vertikalbeschleunigung eines mechanischen Systems bzw. dessen Schwerpunktes in einem solchen Schwerefeld unabhängig herauskommt von dessen innerer kinetischer Energie. Nach der von mir versuchten Theorie war aber die Unabhängigkeit der Fallbeschleunigung von der

\footnotetext{
${ }^{2}$ To be sure, I have to admit that in my opinion the most effective argument for why such a theory [a scalar theory of gravity] has to be abandoned rests on the conviction that relativity holds with respect to a much wider group of substitutions than just the linear-orthogonal ones. However, we are not justified to push this argument since we were not able to determine the (most general) group of substitutions which belongs to our gravitational equations.
} 
Horizontalgeschwindigkeit bzw. der inneren Energie eines Systems nicht vorhanden. Dies paßte nicht zu der alten Erfahrung, daß die Körper alle dieselbe Beschleunigung in einem Gravitationsfeld erfahren. Dieser Satz, der auch als Satz über die Gleichheit der trägen und schweren Masse formuliert werden kann, leuchtete mir nun in seiner tiefen Bedeutung ein. Ich wunderte mich im höchsten Grade über sein Bestehen und vermutete, daß in ihm der Schlüssel für ein tieferes Verständnis der Trägheit und Gravitation liegen müsse. An seiner strengen Gültigkeit habe ich auch ohne Kenntnis des Resultates der schönen Versuche von Eötvös, die mir-wenn ich mich richtig erinnere - erst später bekannt wurden, nicht ernsthaft gezweifelt. Nun verwarf ich den Versuch der oben angedeuteten Behandlung des Gravitationsproblems im Rahmer der speziellen Relativitätstheorie als inadäquat. Er wurde offenbar gerade der fundamentalsten Eigenschaft der Gravitation nicht gerecht. [...] Wichtig war zunächst nur die Erkenntnis, daß eine vernünftige Theorie der Gravitation nur von einer Erweiterung des Relativitätsprinzips zu erwarten war. ${ }^{3}$ (Einstein, 2005, pp. 178-179)

Einstein's belief, that scalar theories of gravity are ruled out, placed him - in this respect - in opposition to most of his colleagues, like Nordström, Abraham, Mie, and Laue, who took part in the search for a (special-) relativistic theory of gravity. (Concerning Nordströms theory and the Einstein-Nordström interaction, compare the beautiful discussions by Norton (1992, 1993)). Some of them were not convinced, it seems, by Einstein's inconsistency argument. For example, even after GR was completed, Laue wrote a comprehensive review paper on Nordströms theory, thereby at least implicitly claiming inner consistency (Laue, 1917). Remarkably, this paper of Laue's is not contained in his collected writings.

On the other hand, modern commentators seem to be content with a discussion of the key rôle that Einstein's arguments undoubtedly played in the development of GR and, in particular, the requirement of general covariance. In fact, already in his famous Vienna lecture (Klein et al., 1995, Doc. 17) held on September 23rd 1913, less than half a year after the submission of the Entwurf Paper, Einstein admits the possibility to sidestep the energyviolation argument given in the latter, if one drops the relation between space-time distances as given by the Minkowski metric on one hand, and physically measured times and lengths on the other. Einstein distinguishes between 'coordinate distances' (German original: 'Koordinatenabstand'), measured by the Minkowski metric, and 'natural distances' (German original: 'natürliche Abstände'), as measured by rods and clocks (Klein et al., 1995, Doc. 17, p. 490). The relation between these two notions of distance is that of a conformal equivalence for the underlying metrics, where the 'natural' metric is obtained from the Minkowski metric by multiplying it with a factor that is proportional to

\footnotetext{
${ }^{3}$ These investigations, however, led to a result which raised my strong suspicion. According to classical mechanics, the vertical acceleration of a body in a vertical gravitational field is independent of the horizontal component of its velocity. Hence in such a gravitational field the vertical acceleration of a mechanical system, or of its centre of gravity, comes out independently of its internal kinetic energy. But in the theory I advanced, the acceleration of a falling body was not independent of its horizontal velocity or the internal energy of the system. This did not fit with the old experience that all bodies experience the same acceleration in a gravitational field. This statement, which can be formulated as theorem on the equality of inertial and gravitational mass, became clear to me in all its deeper meaning. I wondered to the highest degree as to why it should hold and conjectured that it be the key for a deeper understanding of inertia and gravitation. I did not question its rigorous validity, even without knowing about the beautiful experiments by Eötvös, of which - if I remember correctly - I became aware only later. I now abandoned my attempt as inadequate to address the problem of gravitation along the lines outlined above. It obviously could not account for the most fundamental property of gravitation. [...] The important insight at this stage was that a reasonable theory of gravitation could only be expected from an extension of the principle of relativity.
} 
the square of the scalar gravitational potential. Accordingly, the re-publication in January 1914 of the Entwurf Paper includes additional comments, the last one of which acknowledges this possibility to sidestep the original argument against special-relativistic scalar theories of gravity (Klein et al., 1995, Doc. 26, p. 581). This is sometimes interpreted as a 'retraction' by Einstein of his earlier argument (Klein et al., 1995, Doc. 13, p. 342, editors comment [42]) though Einstein himself speaks more appropriately of 'evading' or 'sidestepping' (German original: 'entgehen'). In fact, Einstein does not say that his original argument was erroneous, but rather points out an escape route that effectively changes the hypotheses on which it was based. Indeed, Einstein's re-interpretation of space-time distances prevents the Poincaré transformations from being isometries of space-time, though they formally remain symmetries of the field equations. The new interpretation therefore pushes the theory outside the realm of SR. Hence Einstein's original claim, that a specialrelativistic scalar theory of gravity is inconsistent, is not withdrawn by that re-interpretation. Unfortunately, Einstein's recollections do not provide sufficient details to point towards a unique theory against which his original claim may be tested. But guided by Einstein's remarks and simple first principles one can write down a special-relativistic scalar theory and check whether it really suffers from the shortcomings of the type mentioned by Einstein. This we shall do in the main body of this paper. We shall find that, as far as its formal consistency is concerned, the theory is much better behaved than suggested by Einstein. We end by suggesting another rationale (than violation of energy conservation), which is also purely intrinsic to the theory discussed here, for going beyond Minkowski geometry.

\section{Scalar gravity}

In this section we show how to construct a special-relativistic theory for a scalar gravitational field, $\Phi$, coupled to matter. Before we will do so in a systematic manner, using variational methods in form of a principle of stationary action, we will mention the obvious first and naive guesses for a Poincaré invariant generalisation of formulae (1) and point out their deficiencies.

Our conventions for the Minkowski metric are 'mostly minus', that is, $\eta_{\mu v}=\operatorname{diag}(1,-1,-1,-1)$. Given a worldline, $x^{\mu}(\lambda)$, where $\lambda$ is some arbitrary parameter, its derivative with respect to its eigentime, $\tau$, is written by an overdot, $\dot{x}^{\mu}:=\mathrm{d} x^{\mu} / \mathrm{d} \tau$, where $\mathrm{d} \tau:=c^{-1} \sqrt{\eta_{\mu v}\left(\mathrm{~d} x^{\mu} / \mathrm{d} \lambda\right)\left(\mathrm{d} x^{v} / \mathrm{d} \lambda\right)} \mathrm{d} \lambda . c$ denotes the velocity of light in vacuum (which we do not set equal to unity).

\subsection{First guesses and a naive theory}

There is an obvious way to generalise the left hand side of (1a), namely to replace the Laplace operator by minus (due to our 'mostly minus' convention) the d'Alembert operator:

$$
\begin{aligned}
& \Delta:=\frac{\partial^{2}}{\partial x^{2}}+\frac{\partial^{2}}{\partial y^{2}}+\frac{\partial^{2}}{\partial z^{2}}, \\
& \mapsto \quad-\square:=\frac{\partial^{2}}{\partial x^{2}}+\frac{\partial^{2}}{\partial y^{2}}+\frac{\partial^{2}}{\partial z^{2}}-\frac{1}{c^{2}} \frac{\partial^{2}}{\partial t^{2}}=-\eta^{\mu \nu} \frac{\partial^{2}}{\partial x^{\mu} \partial x^{v}} .
\end{aligned}
$$

This is precisely what Einstein reported: 
Einstein Quote 3. Das einfachste war natürlich, das Laplacesche skalare Potential der Gravitation beizubehalten und die Poisson Gleichung durch ein nach der Zeit differenziertes Glied in naheliegender Weise so zu ergänzen, daß der speziellen Relativitätstheorie Genüge geleistet wurde. ${ }^{4}$ (Einstein, 2005, p. 177)

Also, the right hand side of (1a) need to be replaced by a suitable scalar quantity ( $\rho$ is not a scalar). In SR the energy density is the 00-component of the energy-momentum tensor $T^{\mu v}$, which corresponds to a mass density $T^{00} / c^{2}$. Hence a sensible replacement for the right-hand side of (1a) is

$$
\rho \quad \mapsto \quad T / c^{2}:=\eta^{\mu v} T_{\mu v} / c^{2},
$$

so that (1a) translates to

$$
\Phi=-\kappa T, \quad \text { where } \kappa:=4 \pi G / c^{2}
$$

The replacement (3) is not discussed in Einstein's 1933 recollections, but mentioned explicitly as the most natural one for scalar gravity in Einstein's part of the Entwurf Paper (Klein et al., 1995, Doc. 13, p. 322) and also in his Vienna lecture (Klein et al., 1995, Doc. 17, p. 491). In both cases he acknowledges Laue as being the one to draw his attention to $T / c^{2}$ as being a natural choice for the scalar potential's source.

The next step is to generalise (1b). With respect to this problem Einstein remarks:

Einstein Quote 4. Auch mußte das Bewegungsgesetz des Massenpunktes im Gravitationsfeld der speziellen Relativitätstheorie angepaßt werden. Der Weg hierfür war weniger eindeutig vorgeschrieben, weil ja die träge Masse eines Körpers vom Gravitationspotential abhängen konnte. Dies war sogar wegen des Satzes von der Trägheit der Energie zu erwarten. ${ }^{5}$ (Einstein, 2005, p. 177)

It should be clear that the structurally obvious choice, ${ }^{6}$

$$
\ddot{x}^{\mu}(\tau)=\eta^{\mu \nu} \nabla_{v} \Phi(x(\tau))
$$

cannot work. Four-velocities are normed,

$$
\eta_{\mu v} \dot{x}^{\mu} \dot{x}^{v}=c^{2} \dot{t}^{2}-\dot{x}^{2}-\dot{y}^{2}-\dot{z}^{2}=c^{2}
$$

so that

$$
\eta_{\mu \nu} \dot{x}^{\mu} \ddot{x}^{v}=0
$$

\footnotetext{
${ }^{4}$ The most simple thing to do was to retain the Laplacian scalar potential and to amend the Poisson equation by a term with time derivative, so as to comply with special relativity.

${ }^{5}$ Also, the law of motion of a mass point in a gravitational field had to be adjusted to special relativity. Here the route was less uniquely mapped out, since the inertial mass of a body could depend on the gravitational potential. Indeed, this had to be expected on grounds of the law of inertia of energy.

${ }^{6}$ Throughout we write $\nabla_{\mu}$ for $\partial / \partial x^{\mu}$.
} 
Hence (5) implies the integrability condition $\dot{x}^{\mu}(\tau) \nabla_{\mu} \Phi(x(\tau))=\mathrm{d} \Phi(x(\tau)) / \mathrm{d} \tau=0$, saying that $\Phi$ must stay constant along the worldline of the particle, with renders (5) physically totally useless. The reason for this failure lies in the fact that we replaced the three independent equations (1b) by four equations. This leads to an over-determination, since the four-velocity still represents only three independent functions, due to the kinematical constraint (6). More specifically, it is the component parallel to the four-velocity $\dot{x}$ of the four-vector equation (5) that leads to the unwanted restriction. The obvious way out it to just retain the part of (5) perpendicular to $\dot{x}$ :

$$
\ddot{x}^{\mu}(\tau)=P^{\mu v}(\tau) \nabla_{v} \Phi(x(\tau)),
$$

where

$$
P^{\mu v}(\tau)=\eta_{\lambda}^{\nu \lambda} P_{\lambda}^{\mu}(\tau) \quad \text { and } \quad P_{v}^{\mu}(\tau):=\delta_{v}^{\mu}-\dot{x}^{\mu}(\tau) \dot{x}_{v}(\tau) / c^{2}
$$

is the one-parameter family of projectors orthogonal to the four-velocity $\dot{x}(\tau)$, one at each point of the particle's worldline. Hence, by construction, this modified equation of motion avoids the difficulty just mentioned. We will call the theory based on (4) and (8) the naive theory. We also note that (8) is equivalent to

$$
\frac{\mathrm{d}}{\mathrm{d} \tau}\left(m(x(\tau)) \dot{x}^{\mu}(\tau)\right)=m(x(\tau)) \eta^{\mu v} \nabla_{v} \Phi(x(\tau)),
$$

where $m$ is a spacetime dependent mass, given by

$$
m=m_{0} \exp \left(\left(\Phi-\Phi_{0}\right) / c^{2}\right) .
$$

Here $m_{0}$ is a constant, corresponding to the value of $m$ at gravitational potential $\Phi_{0}$, e.g., $\Phi_{0}=0$.

We could now work out consequences of this theory. However, before doing this, we would rather put the reasoning employed so far on a more systematic basis as provided by variational principles. This also allows us to discuss general matter couplings and check whether the matter coupling that the field equation (4) expresses is consistent with the coupling to the point particle, represented by the equation of motion (8). This has to be asked for if we wish to implement the equivalence principle in the following form:

Requirement 1 (Principle of universal coupling). All forms of matter (including test particles) couple to the gravitational field in a universal fashion.

We will see that in this respect the naive theory is not quite correct. We stress the importance of coupling schemes, without which there is no logical relation between the field equation and the equation of motion for (test-) bodies. This is often not sufficiently taken into account in discussions of scalar theories of gravity; compare (Bergmann, 1956; Dowker, 1965; Harvey, 1965; Wellner \& Sandri, 1964).

\subsection{A consistent model-theory for scalar gravity}

Let us now employ standard variational techniques to establish Poincaré-invariant equations for the scalar gravitational field, $\Phi$, and for the motion of a test particle, so that the principle of universal coupling is duly taken care of. We start by assuming the field 
equation (4). An action whose Euler-Lagrange equation is (4) is easy to guess ${ }^{7}$ :

$$
S_{\text {field }}+S_{\text {int }}=\frac{1}{\kappa c^{3}} \int \mathrm{d}^{4} x\left(\frac{1}{2} \partial_{\mu} \Phi \partial^{\mu} \Phi-\kappa \Phi T\right),
$$

where $S_{\text {field }}$, given by the first term, is the action for the gravitational field and $S_{\text {int }}$, given by the second term, accounts for the interaction with matter.

To this we have to add the action for the matter, $S_{\text {matter }}$, which we only specify insofar as we assume that the matter consists of a point particle of rest-mass ${ }^{8} m$ and a 'rest' of matter that needs not be specified further for our purposes here. Hence $S_{\text {matter }}=S_{\text {particle }}+S_{\text {rom }}$ (rom $=$ rest of matter), where

$$
S_{\text {particle }}=-m c^{2} \int \mathrm{d} \tau \text {. }
$$

We now invoke the principle of universal coupling to find the particle's interaction with the gravitational field. It must be of the form $\Phi T_{\mathrm{p}}$, where $T_{\mathrm{p}}$ is the trace of the particle's energy-momentum tensor. The latter is given by

$$
T_{\mathrm{p}}^{\mu v}(x)=m c \int \dot{x}^{\mu}(\tau) \dot{x}^{v}(\tau) \delta^{(4)}(x-x(\tau)) \mathrm{d} \tau,
$$

so that the particle's contribution to the interaction term in (11) is

$$
S_{\text {int-particle }}=-m \int \Phi(x(\tau)) \mathrm{d} \tau .
$$

Hence the total action can be written in the following form:

$$
\begin{aligned}
S_{\mathrm{tot}}= & -m c^{2} \int\left(1+\Phi(x(\tau)) / c^{2}\right) \mathrm{d} \tau \\
& +\frac{1}{\kappa c^{3}} \int \mathrm{d}^{4} x\left(\frac{1}{2} \partial_{\mu} \Phi \partial^{\mu} \Phi-\kappa \Phi T_{\text {rom }}\right)+S_{\text {rom }} .
\end{aligned}
$$

By construction, the field equation that follows from this action is (4), where the energy-momentum-tensor refers to the matter without the test particle (the selfgravitational field of a test particle is always neglected). The equations of motion for the test particle then turn out to be

$$
\begin{gathered}
\ddot{x}^{\mu}(\tau)=P^{\mu v}(\tau) \partial_{v} \phi(x(\tau)), \\
\text { where } \quad P^{\mu v}(\tau)=\eta^{\mu v}-\dot{x}^{\mu}(\tau) \dot{x}^{v}(\tau) / c^{2} \\
\text { and } \phi:=c^{2} \ln \left(1+\Phi / c^{2}\right) .
\end{gathered}
$$

Three things are worth remarking at this point:

- The projector $P^{\mu v}$ now appears naturally.

- The difference between (8) and (16) is that in the latter it is $\phi$ rather than $\Phi$ that drives the four acceleration. This (only) difference with the naive theory was imposed upon us

\footnotetext{
${ }^{7}$ Note that $\Phi$ has the physical dimension of a squared velocity, $\kappa$ that of length-over-mass. The pre-factor $1 / \kappa c^{3}$ gives the right-hand side of (11) the physical dimension of an action. The overall signs are chosen according to the general scheme for Lagrangians: kinetic minus potential energy.

${ }^{8} \mathrm{We}$ do not need to indicate the rest mass by an additional subscript 0 , since in the sequel we never need to distinguish between rest- and dynamical mass. From now on $m$ will always refer to rest mass.
} 
by the principle of universal coupling, which, as we have just seen, determined the motion of the test particle. This difference is small for small $\Phi / c^{2}$, since, according to $(16 \mathrm{c}), \phi \approx \Phi\left(1+\Phi / c^{2}+\cdots\right)$. But it becomes essential if $\Phi$ gets close to $-c^{2}$, where $\phi$ diverges and the equations of motion become singular. We will see below that the existence of the critical value $\Phi=-c^{2}$ is not necessarily a deficiency and that it is, in fact, the naive theory which displays an unexpected singular behaviour (cf. Section 4.2).

- The universal coupling of the gravitational field to matter only involves the trace of energy-momentum tensor of the latter. As a consequence of the tracelessness of the pure electromagnetic energy-momentum tensor, there is no coupling of gravity to the free electromagnetic field, like, e.g., a light wave in otherwise empty space. A travelling electromagnetic wave will not be influenced by gravitational fields. Hence this theory predicts no deflection of light-rays that pass the neighbourhoods of stars of other massive objects, in disagreement with experimental observations. Note however that the interaction of the electromagnetic field with other matter will change the trace of the energy-momentum tensor of the latter. For example, electromagnetic waves trapped in a material box with mirrored walls will induce additional stresses in the box's walls due to radiation pressure. This will increase the weight of the box corresponding to an additional mass $\Delta m=E_{\mathrm{rad}} / c^{2}$, where $E_{\mathrm{rad}}$ is the energy of the radiation field. In this sense bound electromagnetic fields do carry weight.

Let us now focus on the equations of motion specialised to static situations. That is, we assume that there exists some inertial coordinate system $x^{\mu}$ with respect to which $\Phi$ and hence $\phi$ are static, i.e., $\nabla_{0} \Phi=\nabla_{0} \phi=0$. We have

Proposition 1. For static potentials (16) is equivalent to

$$
\vec{x}^{\prime \prime}(t)=-\left(1-\beta^{2}(t)\right) \vec{\nabla} \phi(\vec{x}(t))
$$

where here and below we write a prime for $\mathrm{d} / \mathrm{d} t$ and use the standard shorthands $\vec{v}=\vec{x}^{\prime}$, $\vec{\beta}=\vec{v} / c, \beta=\|\vec{\beta}\|$, and $\gamma=1 / \sqrt{1-\beta^{2}}$.

Proof. We write in the usual four-vector component notation: $\dot{x}^{\mu}=c \gamma(1, \vec{\beta})$. Using $\mathrm{d} / \mathrm{d} \tau=$ $\gamma \mathrm{d} / \mathrm{d} t$ and $\mathrm{d} \gamma / \mathrm{d} t=\gamma^{3}\left(\vec{a} \cdot \vec{v} / c^{2}\right)$, we have on one side

$$
\ddot{x}^{\mu}=\gamma^{4}\left(\vec{a} \cdot \vec{\beta}, \vec{a}_{\|}+\gamma^{-2} \vec{a}_{\perp}\right),
$$

with $\vec{a}:=\mathrm{d} \vec{v} / \mathrm{d} t . \vec{a}_{\|}:=\beta^{-2} \vec{\beta}(\vec{\beta} \cdot \vec{a})$ and $\vec{a}_{\perp}:=\vec{a}-\vec{a}_{\|}$are, respectively, the spatial projections of $\vec{a}$ parallel and perpendicular to the velocity $\vec{v}$. On the other hand, we have

$$
\begin{aligned}
& -\dot{x}^{\mu} \dot{x}^{\nu} \nabla_{v} \phi / c^{2}=-\gamma^{2}(\vec{\beta} \cdot \vec{\nabla} \phi)(1, \vec{\beta}), \\
& \eta^{\mu \nu} \nabla_{\nu} \phi=(0,-\vec{\nabla} \phi),
\end{aligned}
$$

so that

$$
\left(\eta^{\mu \nu}-\dot{x}^{\mu} \dot{x}^{\nu} / c^{2}\right) \nabla_{\nu} \phi=-\gamma^{2}\left(\beta \cdot \nabla \phi, \vec{\nabla}_{\|} \phi+\gamma^{-2} \vec{\nabla}_{\perp} \phi\right),
$$


where $\vec{\nabla}_{\|}:=\beta^{-2} \vec{\beta}(\vec{\beta} \cdot \vec{\nabla})$ and $\vec{\nabla}_{\perp}:=\vec{\nabla}-\vec{\nabla}_{\|}$are the projections of the gradient parallel and perpendicular to $\vec{v}$ respectively. Equating (18a) and (18d) results in

$$
\begin{aligned}
& \vec{a} \cdot \vec{\beta}=-\gamma^{-2} \vec{\beta} \cdot \vec{\nabla} \phi, \\
& \vec{a}=-\gamma^{-2} \vec{\nabla} \phi .
\end{aligned}
$$

Since (18e) is trivially implied by (18f), (18f) alone is equivalent to (16) in the static case, as was to be shown.

Einstein's second quote suggests that he also arrived at an equation like (17), which clearly displays the dependence of the acceleration in the direction of the gravitational field on the transversal velocity. We will come back to this in the discussion section.

We can still reformulate (17) so as to look perfectly Newtonian (i.e. $m \vec{a}$ equals a gradient field). This will later be convenient for calculating the periapsis precession (cf. Sections 5.1 and 5.2).

Proposition 2. Let $m$ be the rest-mass of the point particle. Then (17) implies

$$
m \vec{a}=-\vec{\nabla} \tilde{\phi}(\vec{x}(t)) \quad \text { with } \tilde{\phi}:=\left(m c^{2} / 2\right) \gamma_{0}^{-2} \exp \left(2 \phi / c^{2}\right),
$$

where $\gamma_{0}$ is an integration constant.

Proof. Scalar multiplication of (17) with $\vec{v}$ leads to

$$
\left(\ln \gamma+\phi / c^{2}\right)^{\prime}=0
$$

which integrates to

$$
\gamma=\gamma_{0} \exp \left(-\phi / c^{2}\right)
$$

where $\gamma_{0}$ is a constant. Using this equation to eliminate the $\gamma^{-2}$ on the right-hand side of (17) the latter assumes the form (19).

\section{Free-fall in static homogeneous fields}

We recall that in Quote 2 scalar gravity was accused of violating a particular form of the principle of the universality of free fall, which Einstein called 'the most fundamental property of gravitation'. In this section we will investigate the meaning and correctness of this claim in some detail. It will be instructive to compare the results for the scalar theory with that of a vector theory in order to highlight the special behaviour of the former, which, in a sense explained below, is just opposite to what Einstein accuses it of. We also deal with the naive scalar theory for comparison and also to show aspects of its singular behaviour that we already mentioned above.

\subsection{The scalar model-theory}

Suppose that with respect to some inertial reference frame with coordinates $(c t, x, y, z)$ the gravitational potential $\phi$ depends only on $z$. Let at time $t=0$ a body be released at the origin, $x=y=z=0$, with proper velocity $\dot{y}_{0}=\dot{z}_{0}=0, \dot{x}_{0}=c \beta \gamma$, and $\dot{t}_{0}=\gamma$ (so as to obey (6)). As usual $c \beta=v=\dot{x}_{0} / \dot{t}_{0}$ is the ordinary velocity and $\gamma:=1 / \sqrt{1-\beta^{2}}$. We take the 
gravitational field to point into the negative $z$ direction so that $\phi$ is a function of $z$ with positive derivative $\phi^{\prime}$. Note that $\dot{z}\left(\phi^{\prime} \circ z\right)=\mathrm{d}(\phi \circ z) / \mathrm{d} \tau$ for which we simply write $\dot{\phi}$ with the usual abuse of notion (i.e. taking $\phi$ to mean $\phi \circ z$ ). Finally, we normalise $\phi$ such that $\phi(z=0)=0$.

The equations of motion (16a) now simply read

$$
\begin{aligned}
\ddot{t} & =-\dot{t} \dot{\phi} / c^{2}, \\
\ddot{x} & =-\dot{x} \dot{\phi} / c^{2}, \\
\ddot{y} & =-\dot{y} \dot{\phi} / c^{2}, \\
\ddot{z} & =-\left(1+\dot{z}^{2} / c^{2}\right) \phi^{\prime} .
\end{aligned}
$$

The first integrals of the first three equations, keeping in mind the initial conditions, are

$$
(\dot{t}(\tau), \dot{x}(\tau), \dot{y}(\tau))=(1, c \beta, 0) \gamma \exp \left(-\phi(z(\tau)) / c^{2}\right) .
$$

Further integration requires the knowledge of $z(\tau)$, that is, the horizontal motion couples to the vertical one if expressed in proper time. ${ }^{9}$ Fortunately, the vertical motion does not likewise couple to the horizontal one, that is, the right-hand side of (22d) just depends on $z(\tau)$. Writing it in the form

$$
\frac{\ddot{z} \dot{z} / c^{2}}{1+\dot{z}^{2} / c^{2}}=-\dot{\phi} / c^{2}
$$

immediately allows integration. For $\dot{z}(\tau=0)=0$ and $\phi(z=h)=0$ (so that $\phi(z<h)<0$ ) we get

$$
\dot{z}=-c \sqrt{\exp \left(-2 \phi / c^{2}\right)-1} .
$$

From this the eigentime $\tau_{h}$ for dropping from $z=0$ to $z=-h$ with $h>0$ follows by one further integration, showing already at this point its independence of the initial horizontal velocity.

Here we wish to be more explicit and solve the equations of motion for the oneparameter family of solutions to (4) for $T=0$ and a $\Phi$ that just depends on $z$, namely $\Phi=g z$, for some constant $g$ that has the physical dimension of an acceleration. As already announced we normalise $\Phi$ such that $\Phi(z=0)=0$. These solutions correspond to what one would call a 'homogeneous gravitational field'. But note that these solutions are not globally regular since $\phi=c^{2} \ln \left(1+\Phi / c^{2}\right)=c^{2} \ln \left(1+g z / c^{2}\right)$ exists only for $z>-c^{2} / g$ and it is the quantity $\phi$ rather than $\Phi$ that corresponds to the Newtonian potential (i.e. whose negative gradient gives the local acceleration).

Upon insertion of $\Phi=g z,(25)$ can be integrated to give $z(\tau)$. Likewise, from (25) and (23) we can form $\mathrm{d} z / \mathrm{d} t=\dot{z} / \dot{t}$ and $\mathrm{d} z / \mathrm{d} x=\dot{z} / \dot{x}$ which integrate to $z(t)$ and $z(x)$, respectively. The results are

$$
\begin{aligned}
& z(\tau)=-\frac{c^{2}}{g}\left\{1-\sqrt{1-(\tau g / c)^{2}}\right\}, \\
& z(t)=-\frac{2 c^{2}}{g} \sin ^{2}(g t / 2 \gamma c), \\
& z(x)=-\frac{2 c^{2}}{g} \sin ^{2}\left(g x / 2 \beta \gamma c^{2}\right) .
\end{aligned}
$$

\footnotetext{
${ }^{9}$ In terms of coordinate time the horizontal motion decouples: $\mathrm{d} x / \mathrm{d} t=\dot{x} / \dot{t}=c \beta \Rightarrow x(t)=c \beta t$.
} 
For completeness we mention that direct integration of (23) gives for the other component functions, taking into account the initial conditions $t(0)=x(0)=y(0)=0$ :

$$
(t(\tau), x(\tau), y(\tau))=(1, c \beta, 0)(\gamma c / g) \sin ^{-1}(g \tau / c) .
$$

The relation between $\tau$ and $t$ is

$$
\tau=(c / g) \sin (g t / \gamma c) \text {. }
$$

Inversion of (26a) and (26b) leads, respectively, to the proper time, $\tau_{h}$, and coordinate time, $t_{h}$, that it takes the body to drop from $z=0$ to $z=-h$ :

$$
\begin{aligned}
\tau_{h} & =\frac{c}{g} \sqrt{1-\left(1-g h / c^{2}\right)^{2}} \approx \sqrt{2 h / g}, \\
t_{h} & =\gamma \frac{2 c}{g} \sin ^{-1}\left(\sqrt{g h / 2 c^{2}}\right) \approx \gamma \sqrt{2 h / g} .
\end{aligned}
$$

The approximations indicated by $\approx$ refer to the leading order contributions for small values of $g h / c^{2}$ (and any value of $\gamma$ ). The appearance of $\gamma$ in (29b) signifies the quadratic dependence on the initial horizontal velocity: the greater the inertial horizontal velocity, the longer the span in inertial time for dropping from $z=0$ to $z=-h$. This seems to be Einstein's point (cf. Quote 2). In contrast, there is no such dependence in (29a), showing the independence of the span in eigentime from the initial horizontal velocity.

The eigentime for dropping into the singularity at $z=-h=-c^{2} / g$ is $\tau_{*}=c / g$. In particular, it is finite, so that a freely falling observer experiences the singularity of the gravitational field $-\vec{\nabla} \phi$ in finite proper time. We note that this singularity is also present in the static spherically symmetric vacuum solution $\Phi(r)=-G m / r$ to $(4)$, for which $\phi(r)=$ $c^{2} \ln \left(1+\Phi / c^{2}\right)$ exists only for $\Phi>c^{2}$, i.e. $r>G m / c^{2}$. The Newtonian acceleration diverges as $r$ approaches this value from above, which means that stars of radius smaller than that critical value cannot exist because no internal pressure can support the infinite inward pointing gravitational pull. Knowing GR, this type of behaviour does not seem too surprising after all. Note that we are here dealing with a non-linear theory, since the field equations (4) become non-linear if expressed in terms of $\phi$ according to $(16 \mathrm{c})$.

\subsection{The naive scalar theory}

Let us for the moment return to the naive theory, given by (4) and (8). Its equations off motion in a static and homogeneous vertical field are obtained from (22) by setting $\phi=g z$. Insertion into (25) leads to $z(\tau)$. The expressions $z(t)$ and $z(x)$ are best determined directly by integrating $\mathrm{d} z / \mathrm{d} t=\dot{z} / \dot{t}$ using (25) and (27). One obtains

$$
\begin{aligned}
& z(\tau)=-\frac{c^{2}}{g} \ln (\cos (g \tau / c)), \\
& z(t)=-\frac{c^{2}}{g} \ln (\cosh (g t / \gamma c)), \\
& z(x)=-\frac{c^{2}}{g} \ln \left(\cosh \left(g x / \beta \gamma c^{2}\right)\right) .
\end{aligned}
$$


The proper time and coordinate time for dropping from $z=0$ to $z=-h$ are therefore given by

$$
\begin{aligned}
\tau_{h} & =\frac{c}{g} \cos ^{-1}\left(\exp \left(-h g / c^{2}\right)\right) \approx \sqrt{2 h / g}, \\
t_{h} & =\frac{c}{g} \gamma \cosh ^{-1}\left(\exp \left(h g / c^{2}\right)\right) \approx \gamma \sqrt{2 h / g},
\end{aligned}
$$

where $\approx$ gives again the leading order contributions for small $g h / c^{2}{ }^{10}$ The general relation between $\tau$ and $t$ is obtained by inserting (30a) into the expression (23) for $\dot{t}$ and integration:

$$
\tau=\frac{2 c}{g}\left\{\tan ^{-1}(\exp (g t / \gamma c))-\pi / 4\right\} .
$$

Note that (31a) is again independent of the initial horizontal velocity, whereas (31b) again is not. Moreover, the really surprising feature of (31a) is that $\tau_{h}$ stays finite for $h \rightarrow \infty$. In fact, $\tau_{\infty}=c \pi / 2 g$. So even though the solution $\phi(z)$ is globally regular, the solution to the equations of motion is in a certain sense not, since the freely falling particle reaches the 'end of spacetime' in finite proper time. This is akin to 'timelike geodesic incompleteness', which indicates singular space-times in GR. Note that it need not be associated with a singularity of the gravitational field itself, except perhaps for the fact that the very notion of an infinitely extended homogeneous field is itself regarded as unphysical.

\subsection{Vector theory}

For comparison it is instructive to look at the corresponding problem in a vector (spin 1) theory, which we here do not wish to discuss in detail. It is essentially given by Maxwell's equations with appropriate sign changes to account for the attractivity of like 'charges' (here masses). This causes problems, like that of runaway solutions, due to the possibility to radiate away negative energy. But the problem of free fall in a homogeneous gravitoelectric field can be addressed, which is formally identical to that of free fall of a charge $e$ and mass $m$ in a static and homogeneous electric field $\vec{E}=-E \vec{e}_{z}$. So let us first look at the electrodynamical problem.

The equations of motion (the Lorentz force law) are

$$
m \ddot{z}^{\mu}=e \eta^{\mu v} F_{v \lambda} \dot{z}^{\lambda},
$$

where $F_{03}=-F_{30}=-E / c$ and all other components vanish. Hence, writing

$$
\mathscr{E}:=e E / m c
$$

we have

$$
\begin{aligned}
& c \ddot{t}=-\mathscr{E} \dot{z}, \\
& \ddot{x}=0, \\
& \ddot{y}=0, \\
& \ddot{z}=-\mathscr{E} c \dot{t} .
\end{aligned}
$$

\footnotetext{
${ }^{10}$ To see this use the identity $\cos ^{-1}(x)=\tan ^{-1}\left(\sqrt{x^{-2}-1}\right)$.
} 
With the same initial conditions as in the scalar case we immediately have

$$
x(\tau)=c \beta \gamma \tau, \quad y(\tau)=0 .
$$

Eqs. (35a) and (35d) are equivalent to

$$
(c \ddot{t} \pm \ddot{z})=\mp \mathscr{E}(c \dot{t} \pm \dot{z})
$$

which twice integrated lead to

$$
\operatorname{ct}(\tau) \pm z(\tau)=A_{ \pm} \exp (\mp \mathscr{E} \tau)+B_{ \pm},
$$

where $A_{+}, A_{-}, B_{+}$, and $B_{-}$are four constants of integration. They are determined by $z(0)=\dot{z}(0)=t(\tau)=0$ and $c \dot{t}^{2}-\dot{x}^{2}-\dot{y}^{2}-\dot{z}^{2}=c^{2}$, leading to

$$
t(\tau)=(\gamma / \mathscr{E}) \sinh (\mathscr{E} \tau)
$$

and also

$$
z(\tau)=-(2 c \gamma / \mathscr{E}) \sinh ^{2}(\mathscr{E} \tau / 2)
$$

Using (39) and (36) to eliminate $\tau$ in favour of $t$ or $x$ respectively in (40a) gives

$$
\begin{aligned}
& z(t)=-\frac{\gamma c}{\mathscr{E}}\left(\sqrt{1+(t \mathscr{E} / \gamma)^{2}}-1\right), \\
& z(x)=-\frac{2 \gamma c}{\mathscr{E}} \sinh ^{2}(x \mathscr{E} / 2 \beta \gamma c) .
\end{aligned}
$$

Inverting (40a) and (40b) gives the expressions for the spans of eigentime and inertial time, respectively, that it takes for the body to drop from $z=0$ to $z=-h$ :

$$
\begin{aligned}
\tau_{h} & =(2 / \mathscr{E}) \sinh ^{-1}(\sqrt{\mathscr{E} h / 2 \gamma c}) \approx \gamma^{-1 / 2} \sqrt{2 h / \mathscr{E} c}, \\
t_{h} & =(\gamma / \mathscr{E}) \sqrt{(1+\mathscr{E} h / \gamma c)^{2}-1} \approx \gamma^{+1 / 2} \sqrt{2 h / \mathscr{E} c} .
\end{aligned}
$$

This is the full solution to our problem in electrodynamics, of which we basically just used the Lorentz force law. It is literally the same in a vector theory of gravity, we just have to keep in mind that the 'charge' $e$ is now interpreted as gravitational mass, which is to be set equal to the inertial mass $m$, so that $e / m=1$. Then $\mathscr{E} c$ becomes equal to the 'gravitoelectric' field strength $E$, which directly corresponds to the strength $g$ of the scalar gravitational field. Having said this, we can directly compare (41) with (29). For small field strength we see that in both cases $t_{h}$ is larger by a factor of $\gamma$ than $\tau_{h}$, which just reflects ordinary time dilation. However, unlike in the scalar case, the eigentime span $\tau_{h}$ also depends on $\gamma$ in the vector case. The independence of $\tau_{h}$ on the initial horizontal velocity is therefore a special feature of the scalar theory.

\subsection{Discussion}

Let us reconsider Einstein's statements in Quote 2, in which he dismisses scalar gravity for it predicting an unwanted dependence on the vertical acceleration on the initial horizontal velocity. As already noted, we do not know exactly in which formal context Einstein derived this result (i.e. what the 'von mir versuchten Theorie' mentioned in Quote 2 actually was), but is seems most likely that he arrived at an equation like (17), 
which clearly displays the alleged behaviour. In any case, the diminishing effect of horizontal velocity onto vertical acceleration is at most of quadratic order in $v / c$.

Remark 1. How could Einstein be so convinced that such an effect did not exist? Certainly there were no experiments at the time to support this. And yet he asserted that such a prediction 'did not fit with the old experience [my italics] that all bodies experience the same acceleration in a gravitational field' (cf. Quote 2). What was it based on?

One way to rephrase/interpret Einstein's requirement is this: the time it takes for a body in free fall to drop from a height $h$ to the ground should be independent of its initial horizontal velocity. More precisely, if you drop two otherwise identical bodies in a static homogeneous vertical gravitational field at the same time from the same location, one body with vanishing initial velocity, the other with purely horizontal initial velocity, they should hit the ground simultaneously.

But that is clearly impossible to fulfil in any special-relativistic theory of gravity, independent of whether it is based on a scalar (or vector) field. The reason is this: suppose $-\nabla^{\mu} \phi=(0,0,0,-g)$ is the gravitational field in one inertial frame. Then it takes exactly the same form in any other inertial frame which differs form the first one by (1) spacetime translations, (2) rotations about the $z$-axis, (3) boosts in any direction within the $x y$-plane. So consider a situation where with respect to an inertial frame, $F$, body 1 and body 2 are simultaneously released at time $t=0$ from the origin, $x=y=z=0$, with initial velocities $\vec{v}_{1}=(0,0,0)$ and $\vec{v}_{2}=(v, 0,0)$, respectively. One is interested whether the bodies hit the ground simultaneously. The 'ground' is represented in spacetime by the hyperplane $z=-h$ and 'hitting the ground' is taken to mean that the word-line of the particle in question intersects this hyperplane. Let another inertial frame, $F^{\prime}$, move with respect to $F$ at speed $v$ along the $x$ axis. With respect to $F^{\prime}$ both bodies are likewise simultaneously released at time $t^{\prime}=0$ from the origin, $x^{\prime}=y^{\prime}=z^{\prime}=0$, with initial velocities $\vec{v}_{1}^{\prime}=(-v, 0,0)$ and $\vec{v}_{2}^{\prime}=(0,0,0)$, respectively, according to the relativistic law of velocity addition. The field is still static, homogeneous, and vertical with respect to $F^{\prime}{ }^{11}$ In $F^{\prime}$ the 'ground' is defined by $z^{\prime}=-h$, which defines the same hyperplane in spacetime as $z=-h$. This is true since $F$ and $F^{\prime}$ merely differ by a boost in $x$-direction, so that the $z$ and $z^{\prime}$ coordinates coincide. Hence 'hitting the ground' has an invariant meaning in the class of inertial systems considered here. However, if 'hitting the ground' are simultaneous events in $F$ they cannot be simultaneous in $F^{\prime}$ and vice versa, since these events differ in their $x$ coordinates. This leads us to the following

Remark 2. Due to the usual relativity of simultaneity, the requirement of 'hitting the ground simultaneously' cannot be fulfilled in any Poincaré invariant theory of gravity.

But there is an obvious reinterpretation of 'hitting the ground simultaneously', which makes perfect invariant sense in SR, namely the condition of 'hitting the ground after the same lapse of eigentime'. As we have discussed in detail above, the scalar theory does indeed fulfil this requirement (independence of $(29 a)$ from $\gamma$ ) whereas the vector theory does not (dependence of (41a) on $\gamma$ ).

\footnotetext{
${ }^{11}$ This is true for gravitational fields that derive from a scalar potential as well as vector potentials. In the scalar case even the strength, $\|\vec{\nabla} \phi\|$, of the field is the same in $F$ and $F^{\prime}$, whereas in the vector case the strength in $F^{\prime}$ is enhanced by a factor $\gamma=1 / \sqrt{1-v^{2} / c^{2}}$. For our argument to work we just need that the field is again static, homogeneous, and vertical. It therefore applies to the scalar as well as the vector case.
} 
Remark 3. The scalar theory is distinguished by its property that the eigentime for free fall from a given altitude does not depend on the initial horizontal velocity.

In general, with regard to this requirement, the following should be mentioned:

Remark 4. Einstein's requirement is (for good reasons) not implied by any of the modern formulations of the (weak) equivalence principle, according to which the world-line of a freely falling test-body (without higher mass-multipole-moments and without charge and spin) is determined by its initial spacetime point and four velocity, i.e. independent of the further constitution of the test body. In contrast, Einstein's requirement relates two motions with different initial velocities.

Finally we comment on Einstein's additional claim in Quote 2, that there is also a similar dependence on the vertical acceleration on the internal energy. This claim, too, does not survive closer scrutiny. Indeed, one might think at first that (17) also predicts that, for example, the gravitational acceleration of a box filled with a gas decreases as temperature increases, due to the increasing velocities of the gas molecules. But this arguments incorrectly neglects the walls of the box which gain in stress due to the rising gas pressure. According to (4) more stress means more weight. In fact, a general argument due to Laue (1911) shows that these effects precisely cancel. This has been lucidly discussed by Norton (1993) and need not be repeated here.

\section{Periapsis precession}

We already mentioned that the scalar theory does not predict any deflection of light in a gravitational field, in violation to experimental results. But in order to stay self contained it is also of interest to see directly that the system given by the field equation (4) and the equation of motion for a test particle (16) violates experimental data. This is the case if applied to planetary motion, more precisely to the precession of the perihelion.

Recall that the Newtonian laws of motion predict that the line of apsides remains fixed relative to absolute space for the motion of a body in a potential with $1 / r$-falloff. Any deviation from the latter causes a rotation of the line of apsides within the orbital plane. This may also be referred to as precession of the periapsis, the orbital point of closest approach to the centre of force, which is called the perihelion if the central body happens to be the Sun. Again we compare the result of our scalar theory with that of the naive scalar theory and also with that of the vector theory. ${ }^{12}$

There exist comprehensive treatments of periapsis precession in various theories of gravity, like (Whitrow \& Morduch, 1965). But rather than trying to figure out which (if any) of these (rather complicated) calculations apply to our theory, at least in a leading order approximation, it turns out to be easier, more instructive, and mathematically more transparent to do these calculations from scratch. A convenient way to compute the periapsis precession in perturbed $1 / r$-potentials is provided by the following proposition, which establishes a convenient and powerful technique for calculating the periapsis precession in a large variety of cases.

\footnotetext{
${ }^{12}$ The scalar theory discussed by Dowker (1965) was just devised to give the correct (i.e. GR-) value for the precession of the periapsis. Since the coupling to matter is not discussed, it makes no statements about light deflection, redshift, etc.
} 
Proposition 3. Consider the Newtonian equations of motion for a test particle of mass $m$ in a perturbed Newtonian potential

$$
U(r)=-\frac{\alpha}{r}+\Delta U(r)
$$

where $\alpha>0$ and $\Delta U(r)$ is the perturbation. The potential is normalised so that it tends to zero at infinity, i.e. $\Delta U(r \rightarrow \infty) \rightarrow 0$. Let $2 \pi+\Delta \varphi$ denote the increase of the polar angle between two successive occurrences of periapsis. Hence $\Delta \varphi$ represents the excess over a full turn, also called the 'periapsis shift per revolution'. Then the first-order contribution of $\Delta U$ to $\Delta \varphi$ is given by

$$
\Delta \varphi=\frac{\partial}{\partial L}\left\{\frac{2 m}{L} \int_{0}^{\pi} r_{*}^{2}(\varphi ; L, E) \Delta U\left(r_{*}(\varphi ; L, E)\right) \mathrm{d} \varphi\right\} .
$$

Here $\varphi \mapsto r_{*}(\varphi ; L, E)$ is the solution of the unperturbed problem (Kepler orbit) with angular momentum $L$ and energy $E$. (As we are interested in bound orbits, we have $E<0$.) It is given by

$$
r_{*}(\varphi ; L, E)=\frac{p}{1+\varepsilon \cos \varphi}
$$

where

$$
\begin{aligned}
& p:=\frac{L^{2}}{m \alpha}, \\
& \varepsilon:=\sqrt{1+\frac{2 E L^{2}}{m \alpha^{2}}} .
\end{aligned}
$$

Note that the expression in curly brackets on the right-hand side of (43) is understood as function of $L$ and $E$, so that the partial differentiation is to be taken at constant $E$.

Proof. In the Newtonian setting, the conserved quantities of energy and angular momentum for the motion in a plane coordinatised by polar coordinates, are given by

$$
\begin{aligned}
& E=\frac{1}{2} m\left(r^{\prime 2}+r^{2} \varphi^{\prime 2}\right)+U(r), \\
& L=m r^{2} \varphi^{\prime},
\end{aligned}
$$

where a prime represents a $t$-derivative. Eliminating $\varphi^{\prime}$ in (45) via (46) and also using (46) to re-express $t$-derivatives in terms of $\varphi$-derivatives, we get

$$
\frac{L^{2}}{m^{2} r^{4}}\left((\mathrm{~d} r / \mathrm{d} \varphi)^{2}+r^{2}\right)=2 \frac{E-U}{m} .
$$

This can also be written in differential form,

$$
\mathrm{d} \varphi=\frac{ \pm \mathrm{d} r L / r^{2}}{\sqrt{2 m(E-U(r))-L^{2} / r^{2}}},
$$

whose integral is just given by (44). 
Now, the angular change between two successive occurrences of periapsis is twice the angular change between periapsis, $r_{\min }$, and apoapsis, $r_{\max }$ :

$$
\begin{aligned}
\Delta \varphi+2 \pi & =2 \int_{r_{\min }}^{r_{\max }} \frac{\mathrm{d} r L / r^{2}}{\sqrt{2 m(E-U(r))-L^{2} / r^{2}}} \\
& =-2 \frac{\partial}{\partial L}\left\{\int_{r_{\min }}^{r_{\max }} \mathrm{d} r \sqrt{2 m(E-U(r))-L^{2} / r^{2}}\right\},
\end{aligned}
$$

where the term in curly brackets is considered as function of $L$ and $E$ and the partial derivative is for constant $E$.

Formula (49) is exact. Its sought-after approximation is obtained by writing $U(r)=$ $-\alpha / r+\Delta U(r)$ and expanding the integrand to linear order in $\Delta U$. Taking into account that the zeroth order term just cancels the $2 \pi$ on the left hand side, we get:

$$
\begin{aligned}
\Delta \varphi & \approx \frac{\partial}{\partial L}\left\{2 m \int_{r_{\min }}^{r_{\max }} \frac{\Delta U(r) \mathrm{d} r}{\sqrt{2 m(E+\alpha / r)-L^{2} / r^{2}}}\right\} \\
& \approx \frac{\partial}{\partial L}\left\{\frac{2 m}{L} \int_{0}^{\pi} r_{*}^{2}(\varphi ; L, E) \Delta U\left(r_{*}(\varphi ; L, E)\right) \mathrm{d} \varphi\right\} .
\end{aligned}
$$

In the second step we converted the $r$-integration into an integration over the azimuthal angle $\varphi$. This we achieved by making use of the identity that one obtains from (48) with $U(r)=-\alpha / r$ and $r$ set equal to the Keplerian solution curve $r_{*}(\varphi ; L, M)$ for the given parameters $L$ and $E$. Accordingly, we replaced the integral limits $r_{\min }$ and $r_{\max }$ by the corresponding angles $\varphi=0$ and $\varphi=\pi+\Delta \varphi / 2$, respectively. Since the integrand is already of order $\Delta U$, we were allowed to replace the upper limit by $\varphi=\pi$, so that the integral limits now correspond to the angles for the minimal and maximal radius of the unperturbed Kepler orbit $r_{*}(\varphi ; L, E)$ given by (44a).

Let us apply this proposition to the general class of cases where $\Delta U=\Delta_{2} U+\Delta_{3} U$ with

$$
\begin{aligned}
& \Delta_{2} U(r)=\delta_{2} / r^{2}, \\
& \Delta_{3} U(r)=\delta_{3} / r^{3} .
\end{aligned}
$$

In the present linear approximation in $\Delta U$ the effects of both perturbations to $\Delta \varphi$ simply add, so that $\Delta \varphi=\Delta_{2} \varphi+\Delta_{3} \varphi$. The contributions $\Delta_{2} \varphi$ and $\Delta_{3} \varphi$ are very easy to calculate from (43). The integrals are trivial and give $\pi \delta_{2}$ and $\pi \delta_{3} / p$, respectively. Using (44b) in the second case to express $p$ as function of $L$, then doing the $L$-differentiation, and finally eliminating $L$ again in favour of $p$ using (44b), we get

$$
\begin{aligned}
& \Delta_{2} \varphi=-2 \pi\left[\frac{\delta_{2} / \alpha}{p}\right]=-2 \pi\left[\frac{\delta_{2} / \alpha}{a\left(1-\varepsilon^{2}\right)}\right], \\
& \Delta_{3} \varphi=-6 \pi\left[\frac{\delta_{3} / \alpha}{p^{2}}\right]=-6 \pi\left[\frac{\delta_{3} / \alpha}{a^{2}\left(1-\varepsilon^{2}\right)^{2}}\right],
\end{aligned}
$$

where we also expressed $p$ in terms of the semi-major axis $a$ and the eccentricity $\varepsilon$ via $p=a\left(1-\varepsilon^{2}\right)$, as it is usually done. Clearly this method allows to calculate in a straightforward manner the periapsis shifts for general perturbations $\Delta_{n} U=\delta_{n} / r^{n}$. For example, the case $n=3$ is related to the contribution from the quadrupole moment of the central body. 


\subsection{Scalar model-theory}

All this applies directly to the scalar theory if its equation of motion is written in the Newtonian form (19). The static and rotationally symmetric solution to (4) outside the point source is $\Phi(r)=-G M / r$, so that

$$
\tilde{\phi}(r)=\left(m c^{2} / 2\right) \gamma_{0}^{-2}\left(1-\frac{G M}{r c^{2}}\right)^{2} .
$$

In order to normalize the potential so that it assumes the value zero at spatial infinity we just need to drop the constant term. This leads to

$$
\begin{aligned}
& \alpha=\gamma_{0}^{-2} G M m, \\
& \delta_{2}=\alpha \frac{G M}{2 c^{2}},
\end{aligned}
$$

so that

$$
\Delta \varphi=\Delta_{2} \varphi=-\pi\left[\frac{G M / c^{2}}{a\left(1-\varepsilon^{2}\right)}\right]=-\frac{1}{6} \Delta_{\mathrm{GR}} \varphi,
$$

where $\Delta_{\mathrm{GR}} \varphi$ is the value predicted by GR. Hence scalar gravity leads to a retrograde periapsis precession.

\subsection{Naive scalar theory}

In the naive scalar theory we have $\phi(r)=-G M / r$ in (19) and therefore

$$
\begin{aligned}
\tilde{\phi}(r) & =\left(m c^{2} / 2\right) \gamma_{0}^{-2} \exp \left(-2 G M / c^{2} r\right) \\
& =\left(m c^{2} / 2\right) \gamma_{0}^{-2}\left\{1-2\left(\frac{G M}{c^{2} r}\right)+2\left(\frac{G M}{c^{2} r}\right)^{2}-\frac{4}{3}\left(\frac{G M}{c^{2} r}\right)^{3}+\cdots\right\} .
\end{aligned}
$$

Again we subtract the constant term to normalize the potential so as to assume the value 0 at infinity. Then we simply read off the coefficients $\alpha, \delta_{2}$, and $\delta_{3}$ :

$$
\begin{aligned}
& \alpha=2\left(G M / c^{2}\right)\left(m c^{2} / 2\right) \gamma_{0}^{-2}, \\
& \delta_{2}=2\left(G M / c^{2}\right)^{2}\left(m c^{2} / 2\right) \gamma_{0}^{-2}, \\
& \delta_{3}=-\frac{4}{3}\left(G M / c^{2}\right)^{3}\left(m c^{2} / 2\right) \gamma_{0}^{-2} .
\end{aligned}
$$

Hence we have

$$
\Delta \varphi=\Delta_{2} \varphi+\Delta_{3} \varphi,
$$

where

$$
\begin{aligned}
& \Delta_{2} \varphi=-2 \pi\left[\frac{G M / c^{2}}{a\left(1-\varepsilon^{2}\right)}\right], \\
& \Delta_{3} \varphi=+4 \pi\left[\frac{G M / c^{2}}{a\left(1-\varepsilon^{2}\right)}\right]^{2} .
\end{aligned}
$$


Recall that (52) neglects quadratic and higher order terms in $\Delta U$. If we expand $\Delta U$ in powers of $G M / c^{2} r$, as done in (56), it would be inconsistent to go further than to third order because $\Delta U$ starts with the quadratic term so that the neglected corrections of order $(\Delta U)^{2}$ start with fourth powers in $G M / c^{2} r$. Hence (58) gives the optimal accuracy obtainable with (43). For solar-system applications $G M / c^{2} a$ is of the order of $10^{-8}$ so that the quadratic term (58c) can be safely neglected. Comparison of (58b) with (55) shows that the naive scalar theory gives a value twice as large as that of the consistent model-theory, that is, $-1 / 3$ times the correct value (predicted by GR).

\subsection{Vector theory}

We start from the following

Proposition 4. The equations of motion (33) for a purely 'electric' field, where $F_{0 i}=-F_{i 0}=$ $E_{i} / c$ and all other components of $F_{\mu \nu}$ vanish, is equivalent to

$$
\left(\gamma(t) \vec{x}^{\prime}(t)\right)^{\prime}=c \overrightarrow{\mathscr{E}}(\vec{x}(t)),
$$

where again the prime' denotes $\mathrm{d} / \mathrm{d} t, \gamma(t):=1 / \sqrt{1-\left\|\vec{x}^{\prime}(t)\right\|^{2} / c^{2}}$, and $\overrightarrow{\mathscr{E}}:=e \vec{E} / m c$.

Proof. We have $\mathrm{d} / \mathrm{d} s=\gamma \mathrm{d} / \mathrm{d} t, \mathrm{~d} \gamma / \mathrm{d} t=\gamma^{3}\left(\vec{\beta} \cdot \vec{\beta}^{\prime}\right)$. Now,

$$
\ddot{z}^{\mu}=c \gamma\left(\gamma^{\prime},(\gamma \vec{\beta})^{\prime}\right) \text { and }(e / m) F_{v}^{\mu} \dot{z}^{\nu}=c \gamma(\overrightarrow{\mathscr{E}} \cdot \beta, \overrightarrow{\mathscr{E}}),
$$

so that (33) is equivalent to

$$
\begin{aligned}
& \overrightarrow{\mathscr{E}} \cdot \vec{\beta}=\gamma^{\prime}=\gamma^{3}\left(\vec{\beta} \cdot \vec{\beta}^{\prime}\right), \\
& \overrightarrow{\mathscr{E}}=(\gamma \vec{\beta})^{\prime}=\gamma^{3} \vec{\beta}_{\|}^{\prime}+\gamma \vec{\beta}_{\perp}^{\prime},
\end{aligned}
$$

where $\|$ and $\perp$ refer to the projections parallel and perpendicular to $\vec{\beta}$, respectively. Since (61b) implies (61a), (33) is equivalent to the former.

We apply this to a spherically symmetric field, where $c \overrightarrow{\mathscr{E}}=-\vec{\nabla} \phi$ with $\phi(r)=-G M / r$. This implies conservation of angular momentum, the modulus of which is now given by

$$
L=\gamma m r^{2} \varphi^{\prime} .
$$

Note the explicit appearance of $\gamma$, which, e.g., is not present in the scalar case, as one immediately infers from (17). This fact makes Proposition 3 not immediately applicable. We proceed as follows: scalar multiplication of (59) with $\vec{v}=\vec{x}^{\prime}$ and $m$ leads to the following expression for the conserved energy:

$$
E=m c^{2}(\gamma-1)+U
$$

where $U=m \phi$. This we write in the form

$$
\gamma^{2}=\left(1+\frac{E-U}{m c^{2}}\right)^{2} .
$$

On the other hand, we have

$$
\gamma^{2} \equiv 1+(\beta \gamma)^{2}=1+(\gamma / c)^{2}\left(r^{\prime 2}+r^{2} \varphi^{\prime 2}\right)=1+\frac{L^{2}}{m^{2} c^{2} r^{4}}\left((\mathrm{~d} r / \mathrm{d} \varphi)^{2}+r^{2}\right)
$$


where we used (62) to eliminate $\varphi^{\prime}$ and convert $r^{\prime}$ into $\mathrm{d} r / \mathrm{d} \varphi$, which also led to a cancellation of the factors of $\gamma$. Equating (64a) and (64b), we get

$$
\frac{L^{2}}{m^{2} r^{4}}\left((\mathrm{~d} r / \mathrm{d} \varphi)^{2}+r^{2}\right)=2 \frac{\tilde{E}-\tilde{U}}{m},
$$

where

$$
\begin{aligned}
& \tilde{E}:=E\left(1+E / 2 m c^{2}\right), \\
& \tilde{U}:=U\left(1+E / m c^{2}\right)-U^{2} / 2 m c^{2} .
\end{aligned}
$$

Eq. (65) is just of the form (47) with $\tilde{E}$ and $\tilde{U}$ replacing $E$ and $U$. In particular we have for $U=m \phi=-G M m / r$ :

$$
\tilde{U}(r)=-\frac{\alpha}{r}+\frac{\delta_{2}}{r^{2}}
$$

with

$$
\begin{aligned}
& \alpha=G M m\left(1+E / m c^{2}\right), \\
& \delta_{2}=-\frac{G^{2} M^{2} m}{2 c^{2}} .
\end{aligned}
$$

In leading approximation for small $E / m c^{2}$ we have $\delta_{2} / \alpha \approx-G M / 2 c^{2}$. The advance of the periapsis per revolution can now be simply read off (52a):

$$
\Delta \varphi=\pi\left[\frac{G M / c^{2}}{a\left(1-\varepsilon^{2}\right)}\right]=\frac{1}{6} \Delta_{\mathrm{GR}} \varphi .
$$

This is the same amount as in the scalar model-theory (compare (55)) but of opposite sign, corresponding to a prograde periapsis precession of $1 / 6$ the value predicted by GR.

\section{Energy conservation}

In this section we finally turn to Einsteins argument of the Entwurf Paper concerning energy conservation. From a modern viewpoint, Einstein's claim of the violation of energy conservation seems to fly in the face of the very concept of Poincare invariance. After all, time translations are among the symmetries of the Poincaré group, thus giving rise to a corresponding conserved Noether charge. Its conservation is a theorem and cannot be questioned. The only thing that seems logically questionable is whether this quantity does indeed represent physical energy. So how could Einstein arrive at his conclusion?

\subsection{Einstein's argument}

Einstein first pointed out that the source for the gravitational field must be a scalar built from the matter quantities alone, and that the only such scalar is the trace $T_{\mu}^{\mu}$ of the energy-momentum tensor (as pointed out to Einstein by Laue, as Einstein acknowledges, calling $T_{\mu}^{\mu}$ the 'Laue Scalar'). Moreover, for closed stationary systems, the so-called LaueTheorem (Laue, 1911) for static systems (later slightly generalised to stationary ones) states that the space integral of $T^{\mu v}$ must vanish, except for $\mu=0=v$; hence the space integral of $T_{\mu}^{\mu}$ equals that of $T^{00}$, which means that the total (active and passive) gravitational mass of 
a closed stationary system equals its inertial mass. However, if the system is not closed, the weight depends on the stresses (the spatial components $T^{i j}$ ).

His argument proper is then as follows (compare Fig. 1): consider a box, $B$, filled with electromagnetic radiation of total energy $E$. We idealise the walls of the box to be inwardly perfectly mirrored and of infinite stiffness, so as to be able to support normal stresses (pressure) without suffering any deformation. The box has an additional vertical strut in the middle, connecting top and bottom walls, which supports all the vertical material stresses that counterbalance the radiation pressure, so that the side walls merely sustain normal and no tangential stresses. The box can slide without friction along a vertical shaft whose cross section corresponds exactly to that of the box. The walls of the shaft are likewise idealised to be inwardly perfectly mirrored and of infinite stiffness. The whole system of shaft and box is finally placed in a homogeneous static gravitational field, $\vec{g}$, which points vertically downward. Now we perform the following process. We start with the box being placed in the shaft in the upper position. Then we slide it down to the lower position; see Fig. 2. There we remove the side walls of the box - without any radiation leaking out - such that the sideways pointing pressures are now provided by the shaft walls. The strut in the middle is left in position to further support all the vertical stresses, as before. Then the box together with the detached side walls are pulled up to their original positions; see Fig. 3. Finally the system is reassembled so that it assumes its initial state. Einstein's claim is now that in a very general class of imaginable scalar theories the process of pulling up the parts needs less work than what is gained in energy in letting the box (with side walls attached) down. Hence, he concluded that such theories necessarily violate energy conservation.

Indeed, radiation-plus-box is a closed stationary system in Laue's sense. Hence, the weight of the total system is proportional to its total energy, $E$, which we may pretend to be given by the radiation energy alone since the contributions from the rest masses of the walls will cancel in the final energy balance, so that we may formally set them to zero at this point. Lowering this box by an amount $h$ in a static homogeneous gravitational field of strength $g$ results in an energy gain of $\Delta E=h g E / c^{2}$. So despite the fact that radiation has a traceless energy-momentum tensor, trapped radiation has a weight given by $E / c^{2}$. This is due to the radiation pressure which puts the walls of the trapping box under tension. For each parallel pair of side-walls the tension is just the radiation pressure, which is one-third

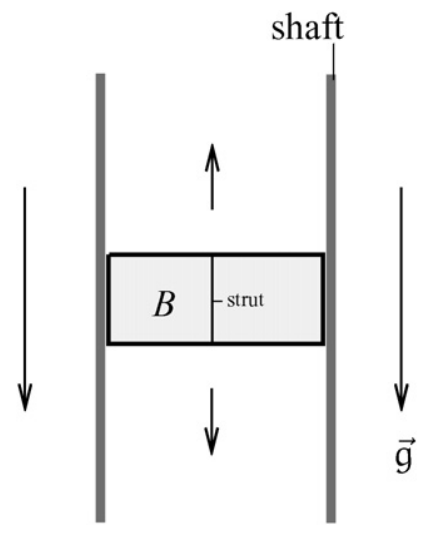

Fig. 1. Sliding box filled with radiation in a gravitational field $\vec{g}$. 


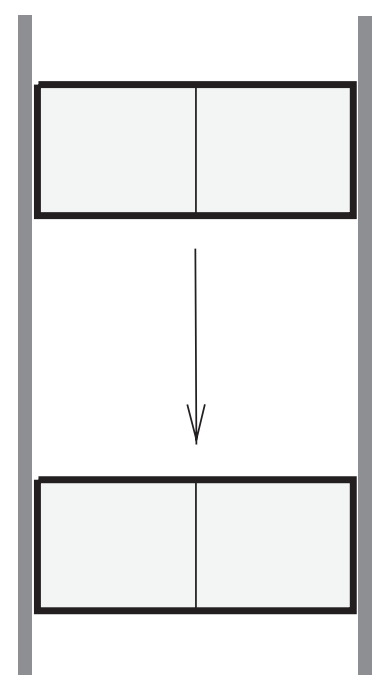

Fig. 2. Lowering the box in the gravitational field with side walls attached.

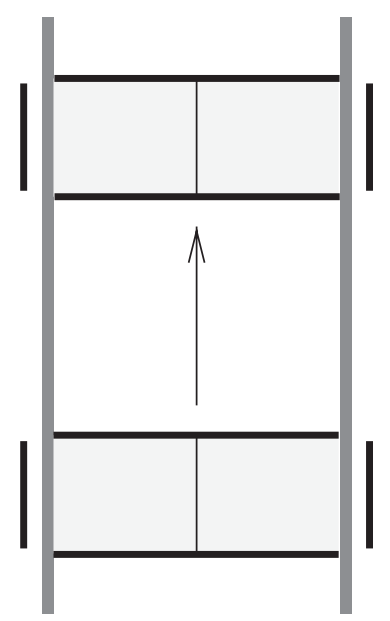

Fig. 3. Raising the box in the gravitational field with side walls taken off.

of the energy density. So each pair of side-walls contribute $E / 3 c^{2}$ to the (passive) gravitational mass (over and above their rest mass, which we set to zero) in the lowering process when stressed, and zero in the raising process when unstressed. Hence, Einstein concluded, there is a net gain in energy of $2 E / 3 c^{3}$ (there are two pairs of side walls).

But it seems that Einstein neglects a crucial contribution to the energy balance. In contrast to the lowering process, the state of the shaft is changed during the lifting process, and it is this additional contribution which just renders Einstein's argument inconclusive. Indeed, when the side walls are first removed in the lower position, the walls of the shaft necessarily come under stress because they now need to provide the horizontal balancing pressures. In the raising process that stress distribution of the shaft is translated upwards. 
But that does cost energy in the theory discussed here, even though it is not associated with any proper transport of the material the shaft is made from. As already pointed out, stresses make their own contribution to weight, independent of the nature of the material that supports them. In particular, a redistribution of stresses in a material immersed in a gravitational field generally makes a non-vanishing contribution to the energy balance, even if the material does not move. This will be seen explicitly below. There seems to be only one paper which explicitly expresses some uneasiness with Einstein's argument, due to the negligence of 'edge effects' (Wellner \& Sandri, 1964, p. 37), however without going into any details, letting alone establishing energy expressions and corresponding balance equations.

\subsection{Energy conservation in the scalar model-theory}

There are 10 conserved currents corresponding to Poincaré-invariance. In particular, the total energy $E$ relative to an inertial system is conserved. For a particle coupled to gravity it is easily calculated and consists of three contributions corresponding to the gravitational field, the particle, and the interaction-energy shared by the particle and the field:

$$
\begin{aligned}
& E_{\text {gravity }}=\frac{1}{2 \kappa c^{2}} \int \mathrm{d}^{3} x\left(\left(\partial_{c t} \Phi\right)^{2}+(\vec{\nabla} \Phi)^{2}\right), \\
& E_{\text {particle }}=m c^{2} \gamma(v), \\
& E_{\text {interaction }}=m \gamma(v) \Phi(\vec{z}(t), t) .
\end{aligned}
$$

Let us return to general matter models and let $T_{\text {total }}^{\mu v}$ be the total stress-energy tensor of the gravity-matter-system. It is the sum of three contributions:

$$
T_{\text {total }}^{\mu v}=T_{\text {gravity }}^{\mu v}+T_{\text {matter }}^{\mu v}+T_{\text {interaction }}^{\mu v},
$$

where $^{13}$

$$
\begin{aligned}
& T_{\text {gravity }}^{\mu v}=\frac{1}{\kappa c^{2}}\left(\partial^{\mu} \Phi \partial^{v} \Phi-\frac{1}{2} \eta^{\mu v} \partial_{\lambda} \Phi \partial^{\lambda} \Phi\right), \\
& T_{\text {matter }}^{\mu v}=\text { depending on matter model, } \\
& T_{\text {interaction }}^{\mu v}=\eta^{\mu v}\left(\Phi / c^{2}\right) T_{\text {matter }}
\end{aligned}
$$

Energy-momentum-conservation is expressed by

$$
\partial_{\mu} T_{\text {total }}^{\mu v}=F_{\text {external }}^{v},
$$

where $F_{\text {external }}^{v}$ is the four-force of a possible external agent. The 0-component of it (i.e. energy conservation) can be rewritten in the form

$$
\text { external power supplied }=\frac{\mathrm{d}}{\mathrm{d} t} \int_{D} \mathrm{~d}^{3} x T_{\text {total }}^{00}+\int_{\partial D} T_{\text {total }}^{0 k} n_{k} \mathrm{~d} \Omega,
$$

for any bounded spatial region $D$. If the matter system is itself of finite spatial extent,

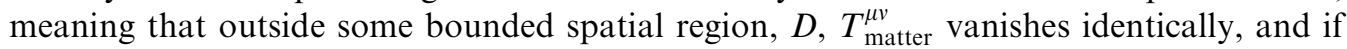
we further assume that no gravitational radiation escapes to infinity, the surface integral in

\footnotetext{
${ }^{13}$ We simply use the standard expression for the canonical energy-momentum tensor, which is good enough in the present case. If $S=\int L \mathrm{~d} t \mathrm{~d}^{3} x$, it is given by $T_{v}^{\mu}:=\left(\partial L / \partial \Phi_{, \mu}\right) \Phi_{, v}-\delta_{v}^{\mu} L$, which here (generally for scalar fields) gives rise to a symmetric tensor, $T^{\mu v}=T^{v \mu}$.
} 
(74) vanishes identically. Integrating (74) over time we then get

$$
\text { external energy supplied }=\Delta E_{\text {gravity }}+\Delta E_{\text {matter }}+\Delta E_{\text {interaction }},
$$

with

$$
E_{\text {interaction }}=\int_{D} \mathrm{~d}^{3} x\left(\Phi / c^{2}\right) T_{\text {matter }},
$$

and where $\Delta$ (something) denotes the difference between the initial and final value of 'something'. If we apply this to a process that leaves the internal energies of the gravitational field and the matter system unchanged, for example a processes where the matter system, or at least the relevant parts of it, are rigidly moved in the gravitational field, like in Einstein's Gedankenexperiment of the 'radiation-shaft-system', we get

$$
\text { external energy supplied }=\Delta\left\{\int_{D} \mathrm{~d}^{3} x\left(\Phi / c^{2}\right) T_{\text {matter }}\right\} .
$$

Now, my understanding of what a valid claim of energy non-conservation in the present context would be is to show that this equation can be violated. But this is not what Einstein did (compare Conclusions).

If the matter system stretches out to infinity and conducts energy and momentum to infinity, then the surface term that was neglected above gives a non-zero contribution that must be included in (77). Then a proof of violation of energy conservation must disprove this modified equation. (Energy conduction to infinity as such is not in any disagreement with energy conservation; you have to prove that they do not balance in the form predicted by the theory.)

\subsection{Discussion}

For the discussion of Einstein's Gedankenexperiment the term (76) is the relevant one. It accounts for the weight of stress. Pulling up a radiation-filled box inside a shaft also moves up the stresses in the shaft walls that must act sideways to balance the radiation pressure. This lifting of stresses to higher gravitational potential costs energy, according to the theory presented here. This energy was neglected by Einstein, apparently because it is not associated with a transport of matter. He included it in the lowering phase, where the sidewalls of the box are attached to the box and move with it, but neglected them in the raising phase, where the side walls are replaced by the shaft, which does not move. But as far as the 'weight of stresses' is concerned, this difference is irrelevant. What (76) tells us is that raising stresses in an ambient gravitational potential costs energy, irrespectively of whether it is associated with an actual transport of the stressed matter or not. This would be just the same for the transport of heat in a heat-conducting material. Raising the heat distribution against the gravitational field costs energy, even if the material itself does not move.

\section{Conclusion}

From the foregoing I conclude that, taken on face value, neither of Einstein's reasonings that led him to dismiss scalar theories of gravity prior to being checked against experiments are convincing. First, energy - as defined by Noether's theorem-is conserved in our model-theory. Note also that the energy of the free gravitational field is positive definite in this theory. Second, the eigentime for free fall in a homogeneous static gravitational field is 
independent of the initial horizontal velocity. Hence our model-theory serves as an example of an internally consistent theory which, however, is experimentally ruled out. As we have seen, it predicts $-1 / 6$ times the right perihelion advance of Mercury and also no light deflection (not to mention Shapiro time-delay, gravitational red-shift, as well as other accurately measured effects which are correctly described by GR).

The situation is slightly different in a special-relativistic vector theory of gravity (Spin 1, mass 0 ). Here the energy is clearly still conserved (as in any Poincaré invariant theory), but the energy of the radiation field is negative definite due to a sign change in Maxwell's equations which is necessary to make like charges (i.e. masses) attract rather than repel each other. Hence there exist runaway solutions in which a massive particle self-accelerates unboundedly by radiating negative gravitational radiation. Also, the free-fall eigentime now does depend on the horizontal velocity, as we have seen. Hence, concerning these theoretical aspects, scalar gravity is much better behaved.

This leaves the question unanswered why Einstein thought it necessary to give up the identification of Minkowski geometry with the physical geometry, as directly measured with physical clocks and rods (cf. the discussion at the end of Section 2). Einstein made it sound as if this was the only way to save energy conservation. This, as we have seen, is not true. But there may well be other reasons to contemplate more general geometries than that of Minkowski space from considerations of scalar gravity as presented here, merely by looking at the gravitational interaction of models for 'clocks' and 'rods'. A simple such model would be given by an electromagnetically bound system, like an atom, where (classically speaking) an electron orbits a charged nucleus (both modelled as point masses). Place this system in a gravitational field that varies negligibly over the spatial extent of the atom and over the time of observation. The electromagnetic field produced by the charges will be unaffected by the gravitational field (due to its traceless energy-momentum tensor). However, (15) tells us that the dynamics of the particle is influenced by the gravitational field. The effect can be conveniently summarised by saying that the masses of point particles scale by a factor of $1+\Phi / c^{2}=\exp \left(\phi / c^{2}\right)$ when placed in the potential $\phi$. This carries over to quantum mechanics so that atomic length scales, like the Bohr radius (in MKSA units)

$$
a_{0}:=\frac{\varepsilon_{0} h^{2}}{m \pi e^{2}}
$$

and time scales, like the Rydberg period (inverse Rydberg frequency)

$$
T_{R}:=\frac{8 \varepsilon_{0}^{2} h^{3}}{m e^{4}},
$$

change by a factor $\exp \left(-\phi / c^{2}\right)$ due to their inverse proportionality to the electron mass $m$ ( $h$ is Planck's constant, $e$ the electron charge, and $\varepsilon_{0}$ the vacuum permittivity). This means that, relative to the units on which the Minkowski metric is based, atomic units of length and time vary in a way depending on the potential. Transporting the atom to a spacetime position in which the gravitational potential differs by an amount $\Delta \phi$ results in a diminishment (if $\Delta \phi>0$ ) or enlargement (if $\Delta \phi<0$ ) of its size and period relative to Minkowskian units. This effect is universal for all atoms.

The question then arises as to the physical significance of the Minkowski metric. Should we not rather define spacetime lengths by what is measured using atoms? After all, as Einstein repeatedly remarked, physical notions of spatial lengths and times should be 
based on physically constructed rods and clocks which are consistent with our dynamical equations. The Minkowski metric would then merely turn into a redundant structure with no direct observational significance. ${ }^{14}$ From that perspective one may indeed criticise special-relativistic scalar gravity for making essential use of dispensable absolute structures, which eventually should be eliminated, just like in the 'flat-spacetime-approach' to GR; compare (Thirring, 1961) and Section 5.2 in (Giulini \& Straumann, 2006). In view of Quote 1 one might conjecture that this more sophisticated point was behind Einstein's criticism. If so, it is well taken. But physically it should be clearly separated from the other explicit accusations which we discussed here.

\section{Acknowledgements}

I thank two anonymous referees for making various suggestions for improvements and John Norton for asking a question that led to the remarks in the second part of Section 7.

\section{References}

Bergmann, O. (1956). Scalar field theory as a theory of gravitation. I. American Journal of Physics, 24(1), 38-42. Dowker, S. (1965). A scalar theory of gravitation. Proceedings of the Physical Society (London), 85(3), 595-600. Einstein, A. (2005). Mein weltbild. Zürich: Europa Verlag (Reprint of the 1953 first edition).

Giulini, D., \& Straumann, N. (2006). Einstein's impact on the physics of the twentieth century. Studies in the History and Philosophy of Modern Physics, 37, 115-173.

Harvey, A. (1965). Brief review of Lorentz-covariant scalar theories of gravitation. American Journal of Physics, 33(6), 449-460.

Klein, M., et al. (Eds.). (1995). The collected papers of Albert Einstein (Vol. 4). Princeton: Princeton University Press.

Laue, M. (1911). Zur Dynamik der Relativitätstheorie. Annalen der Physik, 35, 524-542.

Laue, M. (1917). Die Nordströmsche Gravitationstheorie. Jahrbuch der Radioaktivität und Elektronik, 14(3), 263-313.

Norton, J. (1992). Einstein, Nordström and the early demise of scalar Lorentz-covariant theories of gravitation. Archive for the History of Exact Sciences, 45, 17-94.

Norton, J. (1993). Einstein and Nordström: Some lesser known thought experiments in gravitation. In J. Earman, M. Janssen, \& J. Norton (Eds.), The attraction of gravitation: New studies in history of general relativityEinstein studies, Vol. 5 (pp. 3-29). Boston: Birkäuser.

Thirring, W. (1961). An alternative approach to the theory of gravitation. Annals of Physics, 16, 96-117.

Wellner, M., \& Sandri, G. (1964). Scalar gravitation. American Journal of Physics, 32(1), 36-39 Erratum, Scalar gravitation. American Journal of Physics ibid, 32(6), 504.

Wheeler, J., \& Feynman, R. (1949). Classical electrodynamics in terms of direct interparticle action. Reviews of Modern Physics, 21(3), 425-433.

Whitrow, G., \& Morduch, G. (1965). Relativistic theories of gravitation: A comparative analysis with particular reference to astronomical tests. Vistas in Astronomy, 6(1), 1-67.

\footnotetext{
${ }^{14}$ Note that we are extrapolating here, since our argument is based on the Lagrangian for the specific context. We have not shown that the Minkowski metric can be eliminated in general. 'Clocks' and 'rods' not based on atomic frequencies and lengths scales are clearly conceivable. Moreover, epistemologically speaking, eliminability in a specific context does not imply unobservability in that very same context. For example, as is well known, the electrodynamic field can be altogether eliminated from the description of the dynamics of interacting charged point-particles (Wheeler \& Feynman, 1949). But, surely, this is not saying that the field of one point particle cannot be measured by another one.
} 